\title{
High Performance Silicon Drift Detectors for Energy Dispersive Spectroscopy
}

Andreas Pahlke, Florian Dams, Reinhard Fojt, Michael Fraczek, Lothar Höllt, Jürgen Knobloch, Natsuki Miyakawa

\section{KETEK GmbH, Munich, Germany}

KETEK is the market leader for Silicon Drift Detectors and offers a large variety of sensors with collimated areas starting at $7 \mathrm{~mm}^{2}$ up to $150 \mathrm{~mm}^{2}$ (active area 12 to $170 \mathrm{~mm}^{2}$ ). All detectors are available in different configurations (FET/CUBE, Beryllium/Polymer windows) and KETEK also offers complete electronics to operate the detectors.

Silicon Drift Detectors are very often used in harsh environments combined with high ambient temperatures of up to $+50{ }^{\circ} \mathrm{C}$ or more. These applications ask for very reliable detectors with highly efficient cooling. KETEK will present the latest detector technology where the cooling capacity is improved by more than $15 \mathrm{~K}$ in case of the VITUS H7, H20, H30 and H50 modules.

Tests were performed at ambient temperatures up to $+50^{\circ} \mathrm{C}$ still achieving chip temperatures down to $55{ }^{\circ} \mathrm{C}$ leading to optimum detector performance for all detector sizes. The maximum Peltier power was in parallel reduced by more than $50 \%$ compared to the current thermoelectric cooler at a given fixed temperature. Long term stability tests revealed that the vacuum integrity of the new SDD modules is given even at permanent storage temperatures of $+80^{\circ} \mathrm{C}$.

The new cooling technology is available with our vacuum encapsulated Beryllium detectors. For low energy applications detecting X-ray lines below $1 \mathrm{keV}$ KETEK offers the new technology in combination with AP3.3 windows. In this case the modules are backfilled with Xenon gas to minimize the heat transportation by gas convection. This configuration is available for the VITUS H7, H20, H30 and $\mathrm{H} 50$ with the cold side temperature being as low as $-60{ }^{\circ} \mathrm{C}$ at $+20^{\circ} \mathrm{C}$ heatsink temperature.

Silicon Drift Detectors manufactured by KETEK reveal excellent low energy performance. Even detection of Lithium (at $53 \mathrm{eV}$ ) with windowless modules has been demonstrated (Figure 1). The energy resolution of the Carbon peak $(277 \mathrm{eV})$ is as good as $42 \mathrm{eV}$.

KETEK has developed a new detector window made of graphenic carbon. Both a Beryllium replacement as well as a low energy polymer window alternative has been tested. The transmission for both types of graphenic carbon windows is superior compared to the established Beryllium and polymer windows.

The Beryllium replacement consists of $1 \mu \mathrm{m}$ graphenic carbon without any support grid. The window itself is light tight and there is no necessity to use any additional light blocking layers such as aluminium. The burst pressure of the new windows has been tested up to 5 bar and a burst pressure of 2 bar is guaranteed. To prove the reliability of the new windows, pressure cycle tests have been performed and first prototypes passed more than 10 million cycles (vacuum to one atmosphere) without any degradation of the window performance. 
The low energy polymer alternative consists of a $150 \mathrm{~nm}$ graphenic carbon layer supported by a silicon grid (Figure 2). Again no additional layers are needed to make the window light tide. Up to 10,000 pressure cycles have been performed without any degradation in window performance.

KETEK also offers all electronics necessary to operate the SDD modules including a state of the art digital pulse processor.

\section{References:}

[1] S. Huebner et al, IEEE Trans. Nucl. Sci. 62(2) (2015), p. 588.

[2] S. Huebner et al, Phys. Status Solidi B 252(11) (2015), p. 2564.

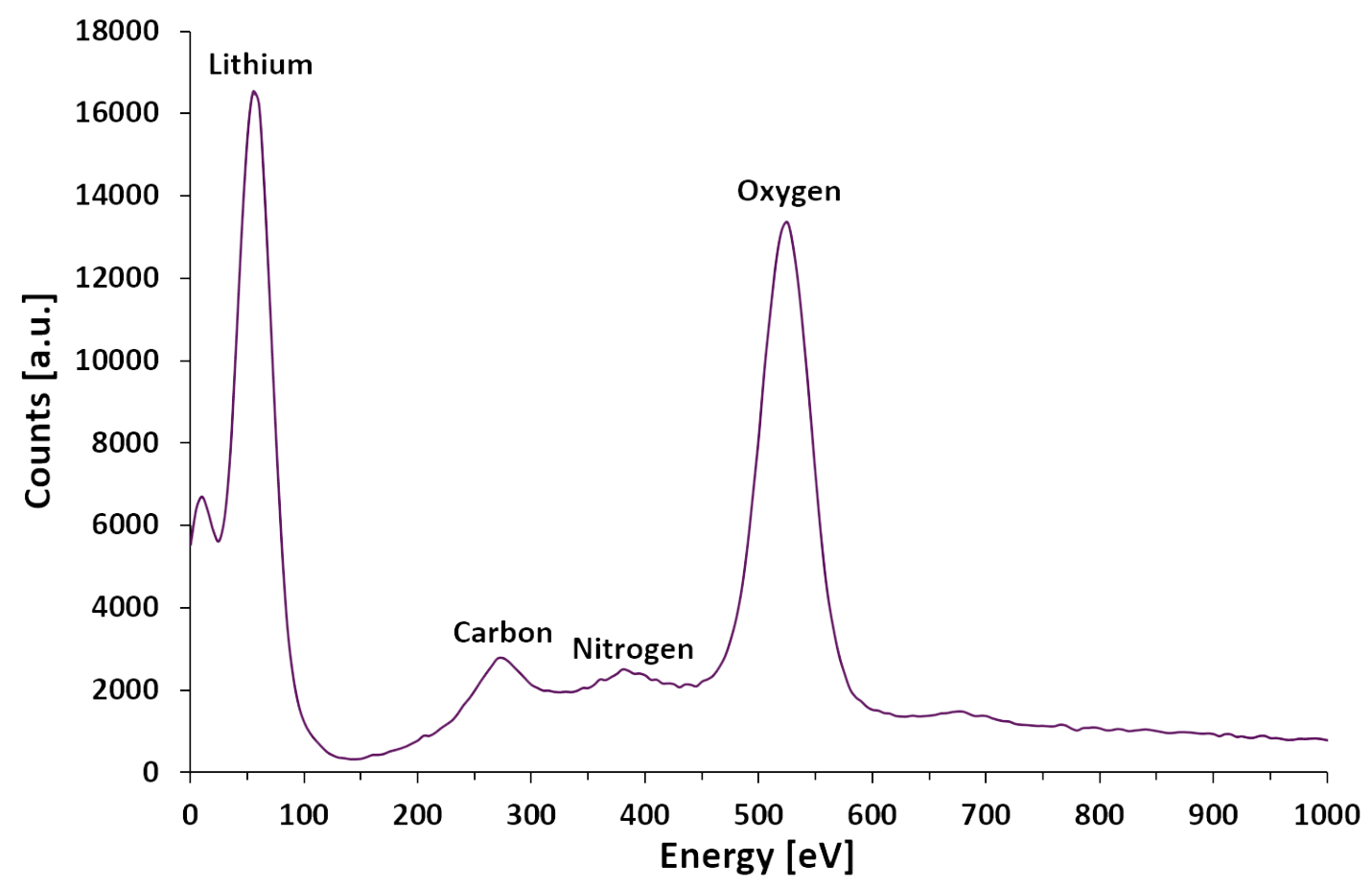

Figure 1. Low energy spectrum of KETEK SDDs showing the Lithium-K $\alpha$ peak
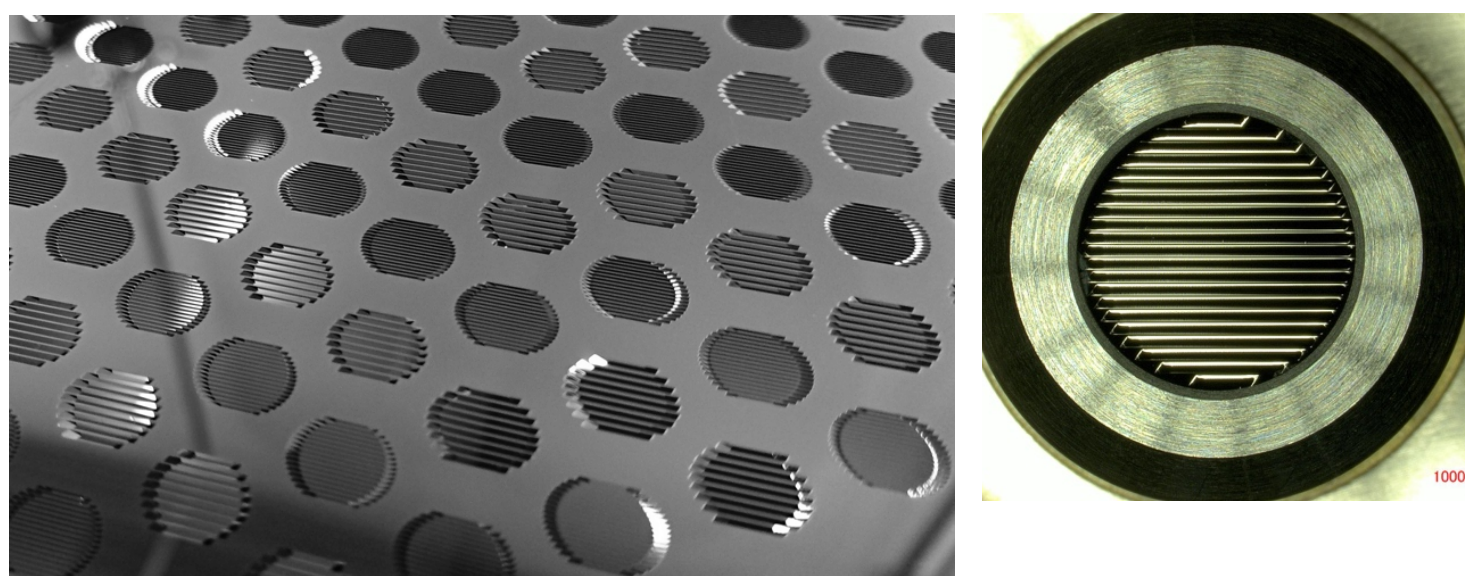

Figure 2. $150 \mathrm{~nm}$ graphenic window mounted on Silicon support grid 